\section{Cahiers de Narratologie}

Analyse et théorie narratives

8 | 1997

Création de l'espace et narration littéraire

\title{
L'espace dans le poème du Rhône de Frédéric Mistral
}

\section{Rémy Gasiglia}

\section{OpenEdition}

Journals

Édition électronique

URL : http://journals.openedition.org/narratologie/10737

DOI : 10.4000/narratologie. 10737

ISSN : 1765-307X

Éditeur

LIRCES

Édition imprimée

Date de publication : 1 décembre 1997

Pagination : 117-130

ISBN : 291089746X

ISSN : 0993-8516

Référence électronique

Rémy Gasiglia, «L'espace dans le poème du Rhône de Frédéric Mistral », Cahiers de Narratologie [En ligne], 8| 1997, mis en ligne le 01 décembre 2020, consulté le 25 février 2021. URL : http:// journals.openedition.org/narratologie/10737 ; DOI : https://doi.org/10.4000/narratologie.10737

Ce document a été généré automatiquement le 25 février 2021.

Article L.111-1 du Code de la propriété intellectuelle. 


\title{
L'espace dans le poème du Rhône de Frédéric Mistral
}

\author{
Rémy Gasiglia
}

1 Les douze chants du Poème du Rhône $e^{1}$ de Mistral racontent le voyage de Lyon à Beaucaire, vers 1830, d'un train de péniches sur lequel prennent place Guilhem, prince d'Orange, fils du roi de Hollande, et une chercheuse d'or nommée l'Anglore. Les deux jeunes gens s'éprennent l'un de l'autre, mais au retour périssent dans le naufrage que provoque un bateau à vapeur. Le poème célébrant la vallée du Rhône, l'espace y est d'abord objet de description. Cependant son importance est telle qu'on doit s'interroger sur son rôle dans la narration et sur ses rapports avec l'écriture.

\section{L'espace comme objet de la description.}

Dans ce texte, où les occurrences du verbe voir ne se comptent plus, l'espace naît des regards. C'est parfois le regard d'un narrateur parlant à la 1ère personne qui transforme certaines descriptions en souvenirs d'enfance et que nous sommes invités à confondre avec Mistral lui-même ${ }^{2}$. Il arrive aussi que ce dernier pratique une focalisation interne à valeur générale, en inventant un observateur anonyme rendu en français par le pronom on et en provençal par une 2ème personne du pluriel qui fait du narrataire le regardant organisateur de la description: " on voit les voiles blanches déferler sous la bise / vesès li vèlo blanco desfourrelado au vènt " (89). Enfin, on rencontre très souvent la focalisation interne classique, dépendant de personnages porte-regard : le batelier Apian surveillant l'appareillage (92), l'Anglore découvrant la foire de Beaucaire (84), le prince d'Orange, touriste étranger justifiant la « description ambulatoire $»^{3}$ des paysages. Dans certains cas leur dialogue assure la description, comme lorsque Apian, «travailleur descripteur $»^{4}$, présente ses barques au prince. Ces focalisations auront des conséquences sur la création de l'espace.

3 Ici, la «topographie » a pour premier principe organisationnel la représentation du mouvement. Cela est sensible au niveau de l'espace textuel. Mistral semble en effet partager les préoccupations de son ami Mallarmé qui, à l'époque de l'écriture du Poème, 
préparait Un coup de dés et selon Claudel méditait sur le « rapport nécessaire entre le contenu poétique et son contenant matériel $»^{5}$. Après avoir lu Le Poème, Mallarmé loua ce « fleuve qui coule selon un livre vivant, chantant et débordant " ${ }^{6}$. Sans doute avait-il été frappé par l'homologie qui y règne entre l'espace de la page et l'espace décrit. L'impression que le fleuve coule selon le livre et que le livre coule comme le fleuve tient d'abord à la succession des cent quatorze laisses en décasyllabes qui reproduit parfaitement le glissement régulier du fleuve et la monotonie du voyage ${ }^{7}$. De plus, la juxtaposition du texte provençal en italique et de sa traduction française en caractères droits fait de chaque page le reflet de sa voisine (certes dans un ordre inverse de celui qu'on attendrait) et instaure entre elles un jeu de miroirs qui rappelle la réflexion des choses par les eaux. L'adéquation fond-forme est encore plus sensible au niveau de la versification et des sonorités. Robert Lafont a montré que les jeux de voyelles des vers non rimés à finale féminine traduisent bien "l'écoulement des eaux" et les "chatoiements de surface $»^{8}$. Mais il affirme que les enjambements sont rares, alors qu'en moyenne un vers sur trois s'enchaîne avec le suivant par un enjambement, un rejet ou un contre-rejet, ce qui contribue à créer une fluidité renforcée par la finale féminine en fin de laisse, aucune strophe n'étant «fermée ». En outre, les liquides et les nasales produisent d'évidents effets d'harmonie imitative. Enfin, les répétitions de formules et de segments narratifs créent d'envoûtants jeux de reflets quasiment aquatiques.

Récit et descriptions renforcent l'impression de mouvement et de miroitement. Le retour régulier de propositions comme "les barques vont glissant» (35) souligne le déplacement des mobiles, tandis que des temps et des auxiliaires d'aspect exprimant l'antériorité font sentir une course plus rapide que la parole des personnages ou du narrateur : " mais les bateaux de maître Apian, rapides, avaient déjà filé sur les eaux fières » (26). Les micro-propositions (adjectifs, groupes nominaux prépositionnels ou relatives) qui situent l'action dans l'espace s'intègrent aux phrases indiquant le mouvement sans en interrompre le flux : « sous la roue du grand soleil et sous le hâle de l'air du Rhône et du vent qui havit, au travers des obstacles de tout genre, des bancs de gravier, des récifs, des gués [...], les Condrillots ${ }^{9}$ avaient pris la remonte » (107). Les séquences descriptives autonomes, narrativisées par le regard des personnages, respectent aussi le dynamisme du texte, Mistral ayant adopté la règle d'écriture suivante : sujet animé + verbe de perception visuelle + complément d'objet (espace à décrire) + autres compléments, comme dans «le vieux patron Apian, [...] en regardant la flotte et la tension des hautes bannes blanches inclinées... » (92). Ce genre de syntagme peut être transformé, sans changement de sens, par mise à la forme passive ou pronominale. Afin de rendre l'illusion d'optique des observateurs mobiles, Mistral attribue le mouvement aux objets fixes grâce à la personnification et applique une seconde règle d'écriture : sujet (espace à décrire personnifié) + verbe d'action + compléments. On aura : "les arcades du Pont Saint-Esprit, prodigieuses, leur passent en triomphe sur la tête " (57). Les deux règles se combinent souvent: «il voit dans l'azur des lagunes se mirer les hauts peupliers qui vont fuyant avec les frênes, les osiers, les digues empierrée » (41). Le mouvement de l'eau et le soleil entraînent des jeux de lumière rendus une fois encore par la personnification ainsi que par l'entrelacement des champs lexicaux comme dans « Au lit du Rhône semé d'îles le soleil jette ses rayonnances tièdes, sur les tourbillons qui tournoient brillants et l'un dans l'autre en bouillonnant se perdent » (40). 
5 En ce qui concerne les volumes, le texte suscite l'illusion d'un espace en trois dimensions. Le point de vue des voyageurs valorise la largeur. L'espace du Rhône est régulièrement délimité à l'aide des prépositions entre (« entre ces falaises » (4), « entre les rives » (7), « entre les bords » (40)) et au milieu de (« au milieu des coteaux parallèles aux rives » (65)). Mais il se dilate (« le fleuve rélargi[t] ses rives» (39), « la plaine [...] s'élargit immense » (94)) et son « amplitude » devient telle que le «vaste fleuve » (91) se transforme en espace épique, « ruche énorme » (3) pour mariniers « colosses » $(1,49)$ aux chevaux "gigantesques" (103). Les descriptions de l'arrière-pays créent la profondeur latérale. Ainsi les voyageurs découvrent-ils dans trois directions et sur trois plans différents le Ventour « au loin », " plus loin encore » le Mézenc des Cévennes, et « là-bas enfin» le Pont Saint-Esprit (43). Parfois, l'horizon se creuse dans une seule direction : c'est la présentation en perspective des Alpes obtenue par le rythme ternaire anaphorique : "et jusqu'au Nivolet de la Savoie, et jusqu'au pic escarpé du Viso, et loin, bien loin, jusqu'à ce mont Genièvre... » (24). L'ocularisation actorielle imposant le respect des lois d'optique, Mistral oppose tel un peintre les couleurs chaudes des premiers plans aux froides des fonds : « aussitôt vues là-haut, [...] les vignes d'or de la Côte-Rotie et aperçus au lointain sur la droite le mont Pilat et ses trois dents bleuâtres... " (19). L'impression de hauteur vient de la description des villes de bas en haut, vues depuis le fleuve. Celle de la Cité des Papes, «colossal entassement de tours ", est fondée sur une nouvelle anaphore qui organise une scénographie de la verticalité : «Avignon sur sa Roque géante ", « Avignon [...] qui [...] élève les pointes de ses clochers », " Avignon [...] que le mistral trousse et décoiffe » (69). La longueur apparaît surtout dans le discours du narrateur qui associe sans cesse le mot «long » au fleuve ${ }^{10}$. Ceci trahit sans doute le point de vue personnel de Mistral qui avait affiché une carte de la vallée du Rhône dans la cage d'escalier de sa maison de Maillane et pouvait avoir une vision " aérienne » du fleuve. Enfin, les embarcations sont en général décrites selon la formule liminaire « de la poupe à la proue » (1), en fonction de la vision d'Apian qui depuis l'arrière du «Caburle » exerce son commandement. A la remontée, le train de bateaux s'allonge du fait du halage par quatre-vingts chevaux. Pour l'étirer, Mistral en a fait un espace sonore. L'ordre de départ, lancé par Apian, relancé en écho par le prouvier et le patron du halage avant d'atteindre le «baile-charretier », allonge la phrase et un espace que le texte désigne avec "dans l'étendue », "déployés en longueur », «les cordages s'étirent » et « la longue file» (93).

Cet espace subjectivisé n'est pas un simple décor. Il a une fonction narrative due à ses rapports avec le temps, les actants et l'action.

\section{L'espace comme moyen de la narration.}

7 Récit de voyage imaginaire, Le Poème du Rhône est fondé sur le chronotope du fleuve navigable. Plus que pour toute route, ici « le temps se déverse dans l'espace et y coule ${ }^{11}$, puisque le lieu de circulation s'écoule avec le temps. Chaque moment de l'action correspondant nécessairement à un point différent de l'espace, chronologie et itinéraire ne font qu'un. Cependant, l'intérêt du texte réside moins dans la temporalité «horizontale» du déroulement syntagmatique des événements narrés que dans la temporalité "verticale ", paradigmatique, liée à l'historicité des lieux décrits. Ainsi, bien des phrases descriptives relevant des deux règles d'écriture se terminent-elles par un complément à valeur temporelle, à l'instar de celle-ci : « [le Prince] aperçoit là-haut, 
qui passent à la file, les châteaux couleur d'or et les tourelles, mémoratifs des époques lointaines» (41). Associant en général à chaque lieu de brefs rappels historiques, ces compléments relèvent de la fonction mathésique. Notons qu'il arrive que le temps «horizontal» et le temps «vertical» coïncident. Ainsi, le temps et l'espace de la diégèse se confondent-ils avec ceux de l'Histoire quand le souvenir de Diane de Poitiers, attaché à Saint-Vallier, s'évanouit derrière des bateaux devenus machines à voyager dans le temps : « Mais Diane est morte, en arrière elle fuit dans le mouvant tableau de ce qui passe autour des nefs qui vont comme des alcyons » (27).

Espace, actants et action sont étroitement associés. D'abord, tous les personnages émanent du même «lieu », le domaine aquatique : les bateliers, du Rhône; Guilhem, des canaux de Hollande (13), l'Anglore, des rives de l'Ardèche. Leur spécificité thématique orientant les choix narratifs, les jeunes gens se rencontrent au confluent du Rhône au nom masculin sur lequel navigue le prince et de l'Ardèche au nom féminin sur les bords de laquelle vit la jeune fille. L'espace aquatique est de plus un véritable actant sur le schéma de Greimas, objet des quêtes. Le prince veut conquérir la vallée, pays de ses ancêtres (16), par le regard et l'esprit ou à travers son allégorie, la Nymphe (12), « la fleur de Rhône » (14), qu'il reconnaît dans l'Anglore (56). Il recherche donc l'eau, parle de s'engloutir dans la fontaine de Tourne (105), puis disparaît dans le Rhône (112). L'Anglore a pour objet tour à tour l'or de l'Ardèche (45), le génie du fleuve appelé le Drac (54), puis le prince auquel elle l'assimile (56), enfin les eaux de la fontaine de Tourne (105) et du fleuve (112). Ensuite, les liens entre l'espace et le reste de la fiction sont tissés au niveau des images par des métaphores intra-diégétiques utilisant l'espace fluvial comme comparant. La vie est donc " une illusion sur l'eau glissante » (71), le temps un "fleuve [...] houleux qui se déroule et fuit » (67), la foule " une houle folle » (84). Réciproquement, l'univers des actants fournit pour décrire l'espace une image qui prend une valeur indicielle: la farandole "d'Arles à Condrieu, aux jours de fête, imitatrice du Rhône en ses détours, ondoie, serpente (en provençal fai la serp, «fait le serpent ») le long de ses berges » (3). La sinuosité du Rhône justifie-t-elle vraiment le rapprochement avec le serpent ? Si l'on dépasse la signification immédiate de la phrase pour en chercher la signifiance ${ }^{12}$, on découvre que le comparant serpent annonce l'Anglore dont le nom signifie "petit lézard gris", le prince confondu par l'Anglore avec le Drac (qui se transforme en serpent), les serpents figurés sur la croix du "Caburle » et sur l'autel mithriaque de Tourne, le Basilic qui pourrait garder le trésor d'Avignon, le vapeur appelé « Le Crocodile » et comparé à un " dragon », le Lert (reptile fantastique) et la tortue géante des grottes de l'Ardèche, et même la Provence, " pays où s'ébat la Tarasque » (autre reptile fantastique) (48). Au niveau structurel, on sait que le mouvement du texte, fondé sur l'aller et retour, dépend du décor spatial et fait du Pont Saint-Esprit le cœur de l'action, lieu de la rencontre à la descente et de la disparition à la remontée de l'Anglore et du Prince $(66-67,112)$. Une autre structure complète celle-là, fondée sur un plan vertical et signifiée métaphoriquement par un leitmotiv relevant de la fonction sémiosique. Il s'agit du signe de croix que, près de la croix de poupe qu'il a fabriquée, Apian dessine à chaque appareillage ${ }^{13}$, organisant et sacralisant l'espace décrit. Car sur le fleuve, les mariniers sont au milieu d'un repère mathématique dont les quatre directions sont sans cesse indiquées: le ciel, les profondeurs des eaux, $l^{\prime}$ «Empire» (ou rive orientale) et le "Royaume» (ou rive occidentale). Si l'ouest et l'est rappellent l'opposition historique France/Saint-Empire, le haut et le bas indiquent l'antithèse religieuse christianisme/paganisme. Le bas est un espace mythique. En effet, le prince qui s'enfonce dans le fleuve avec son aimée rêvait 
de restaurer le culte de Mithra. Dans le Rhône l'Anglore cherchait le Drac et ne pouvait le voir si elle s'était signée avant d'y entrer. D'ailleurs selon Apian cette "masque " "ferait sombrer une barquée de crucifix» (107). Le bas comprend aussi les grottes fantastiques de l'Ardèche et en Avignon un puits mystérieux qui, d'après une voyageuse vénitienne, recèlerait des statues d'or (l'aventurière prétend qu'il s'agit de celles du Christ et des apôtres, mais rien ne le confirme). Espace légendaire, les grottes sont décrites par les personnages $(50-51,96)$ sur le mode merveilleux et le monde subaquatique est peint avec un flou obtenu entre autres par la substantivation des adjectifs. Lumière et couleurs y deviennent des substances, comme " les bleus de l'eau calme» (50). Ces profondeurs sont de plus des métaphores de la sexualité. L'Anglore découvre le désir dans le Rhône, les Vénitiennes qui cherchent le puits au trésor semblent vouloir séduire le prince, celui-ci souhaite passer sa nuit de noces avec l'Anglore dans la fontaine de Tourne. D'ailleurs, le corps féminin est un espace où il est doux de plonger : les femmes de Condrieu « dans la bonne chaleur de leur poitrine forte mettaient la graine des vers à soie couver » (2); la Vénitienne dissimule dans son sein le plan du trésor (70) ; le Drac aime « se glisser sous les jupes de quelque moissonneuse à moitié endormie et, s'entortillant autour de sa taille, l'étreindre doucement de ses circonvolutions et lui téter le sein jusques à ce qu'il tombe assouvi de lait et de volupté » (58). Pour le pieux Apian, le lieu de l'équilibre physique et moral sera le centre de la croix, du bateau et du fleuve : «il faut [...] tenir le milieu », dit-il (30). En sortir entraîne la perturbation qui au niveau narratif suscite le drame. En effet, lorsque les héros quittent le milieu du «Caburle », ils affrontent le malheur. Ne sont-ils pas sur la proue au moment de l'accident? Au début, Apian a raconté l'histoire de "l'insensé » qui brisa d'un coup de fusil un bras du Christ d'un oratoire, qui donc rompit l'équilibre sacré, et dont le bateau se fracassa contre le Pont Saint-Esprit (10). Cette anecdote est à lire comme une mise en abyme prospective et matricielle ${ }^{14}$ de l'énoncé, confirmée par la réitération de l'association du pont et du naufrage $(38,57)$. Il ne s'agit pas du seul cas d'annonce du dénouement à partir de l'espace. On lit à l'incipit la description du saint Nicolas de Condrieu, patron des mariniers, qui dans sa chapelle a "près de lui la cuve d'où l'on voit émerger les têtes des trois mousses réchappés sains et saufs de l'horrible saumure » (2). Cette autre mise en abyme de la catastrophe est suivie d'une dizaine de références au saint ${ }^{15}$ qui rappellent la fatalité pesant sur les voyageurs.

On voit que les éléments textuels renvoyant en apparence à l'espace référentiel jouent un rôle essentiel dans le système narratif. De plus, suscitant la réflexivité au niveau de l'énoncé et, on le verra, participant à la réflexion du code et de l'énonciation, ils révèlent l'activité créatrice consciente de l'auteur et donc la littérarité ${ }^{16}$ d'un texte qui crée surtout un espace de l'écriture.

\section{L'espace de l'écriture.}

10 Car l'espace décrit subit un processus de déréalisation dont le projet est inscrit au cœur de la fiction dans les paroles du prince: «Ne regarde pas dans l'eau - qui est trop profonde, Ne regarde pas la terre - qui est trop loin, Ne regarde pas le ciel - qui est trop vaste : regarde dans mon âme où tu es le soleil !» (65). Emergeant du brouillard au début du poème (8), les bateaux disparaissent à la fin dans les eaux. Entre ces deux points du récit, les compléments à valeur spatiale des syntagmes descriptifs peignent souvent des ciels dont le vide, la limpidité font transition vers l'irréalité de la 
dimension temporelle: "et les clochers et les tours et les temples dans la lumière inondant et limpide écrivent du passé l'histoire auguste » (17). Parfois, l'expansion à valeur temporelle provoque le passage à l'abstraction, comme avec les «vieux châteaux emmantelés de gloire » (27). L'impression d'effacement de l'espace provient également des très nombreuses occurrences dans l'ensemble du texte de l'adjectif «blanc », dont l'effet déréalisant est accru dans la phrase suivante par l'association de la blancheur à l'éloignement : «Et la vue merveilleuse d'Avignon, son grand château aux gigantesques murs, ses remparts crénelés, tours et tourelles, dans le matin blanc de clarté s'éloignent " (78). De même que les couleurs, les formes disparaissent quand l'évocation des crues du Rhône trouble la distinction terre/eau. De plus, le chant central s'intitule "A l'avalido», ce que Mistral traduit par «A horizon perdu » mais qui littéralement signifie «Vers l'anéantissement ». Ne faut-il pas mettre en relation cette néantisation avec la non-action du protagoniste? Le prince, dont les opposants sont incertains ou inefficaces, rêve de «néant » (18), n'achève aucune entreprise et à Avignon interrompt sans raison valable la recherche du trésor. Cet épisode décevant révèle l'abandon de la fiction, dans un poème dont les héros font un "trou dans l'eau » (10) et qui raconte comment l'espace rhodanien est passé de sa merveilleuse animation d'autrefois à son vide présent : « tout cela aujourd'hui est mort, muet et vaste » (3). Devant l'annulation de l'espace et de l'action on peut songer au vers du troubadour Guillaume de Poitiers «Farai un vers de dreit nien» («je ferai un vers de pur néant», ou selon Zumthor «l'objet de ma chanson, c'est un néant $\left.\aleph^{17}\right)$, ainsi qu'à «l'aboli bibelot d'inanité sonore ». Or, le véritable objet de la quête du prince, c'est bien un néant "sonore », ce que révèle l'épisode d'Orange. Guilhem veut découvrir cette ville qu'il ne connaît que par ses lectures (16) mais n'y parviendra pas car elle est invisible depuis le fleuve (78). Il y a là un vide au cœur du texte, un "blanc» dans la série des descriptions qui contraindra le lecteur comme le prince à imaginer cet espace tant désiré. La similitude est grande avec "L'Infinito» («L'Infini») de Giacomo Leopardi (1819), idylle dans laquelle le poète, devant une haie " qui du plus lointain horizon cache au regard une telle étendue ", rêve "des espaces interminables au-delà, de surhumains silences", se souvient " de l'éternel, des saisons qui sont mortes ». «Ainsi, dit-il, dans tant d'immensité ma pensée sombre [s'annega], et m'abîmer [il naufragar] m'est doux en cette mer ${ }^{18}$. Sous le titre "A horizon perdu», Guilhem songe devant l'écran des arbres à "la mémoire » de ses ancêtres. Plus tard, non loin d'Orange, il se noiera lors d'un naufrage qui, au fond, lui sera doux. Même refus de l'espace réel au bénéfice du rêve chez Leopardi et chez Mistral. De la capitale des ancêtres du prince il ne reste qu'un nom, signe sans référent. De l'espace provençal du temps des héros ne subsiste que le mot qui le désignait: " cet empire enfin des Bosonides [...] dans le cri 'empire!' dure encore ». Seul demeure donc l'empire du langage, la poésie. Réactivant le topos de l' ubi sunt, Guilhem lance devant l'espace absent : "'Où êtes-vous, Guibour, vaillante épouse de Guilhem au Court-Nez, et toi, princesse Tibour d'Orange ?' - 'Là derrière, répliquent les braves mariniers, là, derrière les arbres ", c'est-à-dire dans l'espace rêvé, l'espace de la littérature, où perdure le souvenir des héroïnes disparues et des réalités effacées. Selon Mallarmé «tout, au monde, existe pour aboutir à un livre ${ }^{19}$; chez Mistral, le livre est le substitut d'un monde perdu.

11 On ne s'étonnera pas que Le Poème fasse tant de place à la réflexion du code ${ }^{20}, c^{\prime}$ est-àdire ici d'une poétique fondée sur le symbole. Il raconte un voyage dans l'espace des signes : signes de croix, mais aussi croix de poupe aux symboles de la Passion et pierre de Tourne aux symboles mithriaques que détaillent deux ekphrasis ${ }^{21}$. Le corps du 
prince porte des signes, tatouage sur le bras et lignes de la main annonçant son destin. Avec l'or de l'Anglore on fait des bagues sur lesquelles l'on grave des emblèmes, Drac et lézardeau. Le Poème raconte aussi un voyage à travers le langage et les textes qui donnent sa seule réalité à l'espace décrit. Le vrai Rhône est celui du poème, car celui du réel «crève ", cela est dit à l'incipit (dans le sens de « déborde ») et redit à l'explicit (dans le sens de "meurt»), quand il est clair que la batellerie traditionnelle a vécu. L'essence du paysage réside dans les toponymes qui constellent l'espace textuel. Celuici est aussi constitué de morceaux de textes : des locutions dans le jargon des mariniers, leur Pater, leurs chansons, leurs anecdotes et encore la chanson des Vénitiennes, plusieurs fois citée ${ }^{22}$, la fable de Galatée et d'Acis (66), l'histoire d'Aucassin et Nicolette (88). Certains sculptent l'espace de la page par leur métrique ou leurs caractères différents. A travers une intertextualité omniprésente dont il est lui-même issu (personnage-citation, il porte le nom de son ancêtre Guillaume d'Orange, héros de chansons de geste du XIIème siècle ${ }^{23}$ ), Guilhem est en quête de «jolis mots » (66). De ce point de vue, il représente sans doute aussi un peu Mistral et l'espace qu'il traverse dans le Poème permet enfin la réflexion de l'énonciation.

On sait que pour écrire cette œuvre, Mistral a utilisé un portulan ${ }^{24}$. Mettant en abyme la naissance de son écriture par une sorte de réduplication aporistique ${ }^{25}$, il montre Guilhem feuilletant l'ouvrage d'où est issu le texte dont celui-ci est le héros (18). De plus, en insistant à l'ouverture et à la clôture du récit $(8,92)$ sur le travail de l'écrivain de bord qui « enregistre » des marchandises synecdoques de la Provence, il symbolise sa propre écriture, expression de sa « volonté de tout nommer, de tout inventorier » de l'espace provençal ${ }^{26}$. Sans insister sur les métaphores de l'écriture qui parsèment le texte, du topo du destin aux monuments qui « écrivent » l'histoire (17), notons que la fin du poème coïncide avec le silence des personnages, réflexion de celui de l'écrivain : « toute la troupe, [...] remonta vers Condrieu, sans autre plainte (senso mai dire) » (114). Avec «l'amplitude et le silence du vaste Rhône» (109), l'explicit conclut une association progressive de l'immensité de l'espace au vide et au silence qui va jusqu'à la spatialisation de celui-ci («tellement est vaste le silence» (40)) et contraste avec les vertigineuses accumulations de choses et de gens qui emplissent l'espace à Beaucaire. Dans les premiers vers, qui annoncent la fin, Mistral déclare que de la vieille batellerie, de la vieille Provence, il ne reste qu'une "trace», «le sillon que le câble [de halage] a creusé contre les pierres [de la berge] »(3), métaphore de sa propre écriture. Et faut-il s'étonner qu'il situe le début de son récit dans la région lyonnaise ? C'est là que selon lui naît le mistral (2), «bise formidable» certes, mais également "harmonieuse cornemuse " (103), dont il porte le nom. Car l'action se déroule à l'époque de la naissance du poète. Le Poème du Rhône est peut-être le poème de Mistral lui-même, lié comme le vent homonyme à l'espace provençal auquel il a prêté, sa vie durant, son souffle épique. Ce serait le poème-fleuve du poète, qui place dans le fleuve un rêve de poésie absolue, idéale, pure parole inouïe des mortels que murmurent les lèvres blanches du Drac: "de ses lèvres tremblantes et pâles sortaient des mots d'amour mystérieux, dans l'eau se perdant incompréhensibles » (53)...

Dans cette ultime épopée mistralienne, l'espace fait l'objet d'un traitement d'une rare richesse qui se signale par la triple motivation du glissement de la référentialité à la réflexivité. Cela peut être dû à la nature même du texte poétique qui selon Riffaterre n'a de véritable référent externe que textuel ${ }^{27}$. Mais ici l'annulation de l'espace au profit de la célébration du langage et de la poésie pourrait venir aussi du sentiment pessimiste d'un échec du Félibrige, d'une impossible action sur le monde réel que 
traduit Le Poème du Rhône selon Robert Lafont ${ }^{28}$. Mais pourquoi ne pas invoquer enfin le contexte symboliste? Lafont y a recouru pour expliquer la versification de cette œuvre et Ripert pour en évoquer brièvement la "poésie un peu mystérieuse ${ }^{29}$. On le fera à propos de la technique du point de vue, de l'émiettement de la description au fil du récit ${ }^{30}$, de la poétique des symboles et peut-être aussi à propos de l'effacement du décor spatial et des personnages, qui rappelle par exemple Pelléas et Mélisande de Maeterlinck : châteaux croulants, grottes merveilleuses et inquiétantes où l'on n'entre pas, objets d'or insaisissables, naufrages, engloutissement des princes Pelléas et Guilhem ${ }^{31}$ épris de fées des eaux, Anglore-lézard et Mélisande-Mélusine ${ }^{32}$... Il nous semble que ce traitement de l'espace permet de lire Le Poème du Rhône, finalement poème du vide, du silence et des signes, comme une somptueuse contribution provençale au mouvement symboliste.

\section{NOTES}

1. MistRAL, Frédéric : Le Poème du Rhône, en XII chants. Texte provençal et traduction française, Paris, Lemerre, 1897, $351 \mathrm{p}$.

2. Cf. « nous venions, enfants, voir sur l'eau longue, voir passer fiers, les mains au gouvernail, les Condrillots » (3). N.B. : Les numéros entre parenthèses sont ceux des laisses.

3. HAMON, Philippe : Introduction à l'analyse du descriptif, Paris, Hachette, 1981, pp. 189 et 218 ; formule empruntée à RICATTE, Robert : La Création romanesque chez les Goncourt, Paris, A. Colin, 1952, pp. 280 et passim.

4. Selon l'expression de Philippe Hamon (op. cit., pp. 202 sqq.).

5. «Philosophie du livre », in Positions et propositions, cité par BUTOR, Michel : "Sur la page », in Répertoire II, Paris, Les Éditions de Minuit, 1964, pp. 101-102.

6. Cité par LAFONT, Robert : in Mistral ou l'illusion, Valderiès, Vent terral, 1980, p. 266.

7. Cf. RIPERT, Émile : La Versification de Frédéric Mistral, Paris, Champion, Aix-en-Provence, Dragon, s.d., p. 152.

8. «Le Poème du Rhône est bien l'exemplaire le plus complet que puisse nous livrer une littérature européenne d'une correspondance du vers au sujet » (Mistral ou l'illusion, op. cit., p. 269).

9. Les Condrillots : les mariniers sont originaires de Condrieu, localité de la région lyonnaise.

10. Cf. « le Rhône long» (82), « l'eau longue » (3,14 et passim) et des tournures comme «le long du Rhône » (75), « le long du fleuve » (19), « le long de l'eau » (43). Cette dimension s'étend aux bateaux - « la longue flotte » (40), le « long bateau à feu » (109) - et aux rives : « le long quai du port » (89), « le long des oseraies », « le long des digues » (97).

11. BAKHTINE, Mikhail : Esthétique et théorie du roman, Paris, Gallimard, 1978, p. 385.

12. Cf. R IfFATERRE, Michaël : "L'Illusion référentielle", in BARTHES, Roland, HAmon, Philippe, RIFFATERRE, Michaël et al., Littérature et réalité, Éditions du Seuil, s. 1., 1982, pp. 96-97.

13. Cf. $7,9,33,93$.

14. DAllenBACH, Lucien : Le Récit spéculaire. Essai sur la mise en abyme, Paris, Editions du Seuil, 1977, p. 83.

15. Cf. 4, 6, 21, 26, 57, 77, 90, 105, 113. 
16. Cf. DALlenBACH, Lucien : op. cit., pp. 78-79 : « En tant que second signe en effet, la mise en abyme ne met pas seulement en relief les intentions signifiantes du premier (le récit qui la comporte), elle manifeste qu'il ( $\left.n^{\prime}\right)$ est lui aussi (qu')un signe et proclame tel n'importe quel trope - mais avec une puissance décuplée par sa taille : Je suis littérature, moi et le récit qui m'enchâsse ».

17. ZuMTHOR, Paul : La Mesure du monde, Paris, Éditions du Seuil, 1993, p. 378.

18. LEOPARDI, Giacomo : Canti, traductions de F.-A. Aulard, Juliette Bertrand, Philippe Jaccottet et Georges Nicole, Paris, N.R.F.-Gallimard, 1982, p. 68.

19. Mallarme, Stéphane : Divagations, Paris, Charpentier, 1897, p. 273.

20. Cf. DallenBaCh, Lucien : op. cit., pp. 128-131.

21. Cf. 6,61 .

22. Cf. $42,56,74,108$.

23. On aura remarqué qu'il porte également le prénom du plus ancien troubadour dont on ait conservé les oeuvres, Guillaume de Poitiers (1071-1127), cité supra.

24. La Vallée du Rhône de Lyon à la mer, du baron Raverat (1889). Cf. Léon Tessier, in Calendau, $\mathrm{n}^{\circ} 84$, décembre 1940, article cité in LAFONT, Robert : op. cit., p. 270 sqq.

25. Ou « fragment censé inclure l'œuvre qui l'inclut » (DALLENBACH, Lucien : op. cit., p. 51).

26. SoubiRAn, Jean-Roger: Le Paysage provençal et l'école de Marseille avant l'impressionnisme, 1845-1874, Paris, Réunion des musées nationaux, Toulon, Musée de Toulon, 1992, p. 129.

27. Cf. RIFFATERRE, Michaël : op. cit., p. 118.

28. Cf. LAFONT, Robert : « La Cause vaincue », op. cit., pp. 299-304.

29. RIPERT, Emile : op. cit., p. 151.

30. Cf. RAIMOND, Michel : La Crise du roman. Des lendemains du naturalisme aux années vingt, Paris, J. Corti, 1966, pp. 305-306.

31. L'un et l'autre sont des « chevaliers au cygne ». Cf. Le Poème, 12, et CITTI, Pierre : « Préface » à Pelléas et Mélisande de Maurice Maeterlinck, s. 1., Le Livre de Poche, 1989, p. 21.

32. Même si l'univers spatial de Pelléas doit se concevoir en fonction des lois du genre dramatique et si la question de l'espace chez les symbolistes mérite sans doute d'être encore approfondie.

\section{AUTEUR}

\section{RÉMY GASIGLIA}

Université de Nice-Sophia Antipolis 